${ }^{1}$ UCI/UPC Hospital Dr. Gustavo Fricke, Viña del Mar, Chile. Unidad de Medicina Intensiva, Universidad de Valparaíso. 2División de Gestión de Redes Asistenciales, Ministerio de Salud de Chile.

UCI/UPC Hospital Naval Almirante Neff, Viña del Mar, Chile. ${ }^{4}$ Hospital Clínico de la Universidad de Chile, Unidad de Medicina Intensiva. Santiago de Chile.

5Unidad de Medicina Intensiva Pontificia Universidad Católica de Chile. Santiago de Chile.

${ }^{6}$ UCI/UPC Hospital de Coquimbo, Coquimbo.

${ }^{7}$ UCI/UPC Hospital de Curicó, Curicó

${ }^{8}$ Unidad de Medicina Intensiva, Universidad de Chile, Santiago de Chile. 'UCI/UPC Hospital Clínico de la Fuerza Aérea de Chile. Sociedad de Medicina Intensiva de Chile. Santiago de Chile.

${ }^{10}$ Subsecretaría de Redes, Ministerio de Salud de Chile.

Recibido el 1 de octubre de 2012 aceptado el 30 de noviembre de 2012

Correspondencia a: Dr. Sergio Gálvez Gómez. UCI/UPC Hospital Dr Gustavo Fricke Alvarez 1532. Viña del Mar Chile. Teléfono: 032-233.86.27 E-Mail: sergiogalvez@fideco.cl

\section{Medicina intensiva en Chile: desafíos para su desarrollo. Documento de la Comisión Nacional de Medicina Intensiva del Ministerio de Salud}

\author{
SERGIO GÁlVEZ G. ${ }^{1}$, HUGO GONZÁlEZ D. ${ }^{2}$, \\ EDUARDO LABARCA M. ${ }^{3}$, RODRIGO CORNEJO R. ${ }^{4}$, \\ ALEJANDRO BRUHN C. ${ }^{5}$, HÉCTOR UGARTE E. ${ }^{6}$, \\ JORGE CANTEROS G. ${ }^{7}$, EDUARDO TOBAR A. ${ }^{8}$, \\ RODRIGO SOTO F. ${ }^{9}$, LUIS CASTILLO F. ${ }^{10}$
}

\begin{abstract}
Intensive care medicine in Chile is still in its dawn. It has experienced a progressive growth in the last decade, but continues to be weak. Although investments in the discipline have increased fivefold, there is still a severe deficiency of intensive care specialists. This issue will represent a serious problem in the near future. The Ministry of Health gathered an expert committee to study the problem and propose solutions for the future development of the discipline.
\end{abstract}

(Rev Med Chile 2013; 141: 90-94).

Key words: Education, medical, graduate; Intensive care; Public health.
L a Medicina Intensiva es una especialidad joven en Chile, con no más de 25 años de existencia desde su reconocimiento formal. En este período ha hecho una contribución significativa a la salud pública al hacerse cargo de los cuidados de los pacientes graves, de manera organizada y con resultados que van más allá de lo esperado por el crecimiento tecnológico y económico del país. El desempeño de este rol ha permitido el desarrollo y fortalecimiento de otras especialidades que, sin el apoyo de la medicina intensiva, no habrían podido consolidarse ni progresar, como la cirugía de alta complejidad, los trasplantes de órganos sólidos, la hematología y oncología moderna, etc. Por lo mismo, es cada vez menos aceptable la existencia de inequidad en el acceso y calidad a estos servicios, donde el factor geográfico de nuestro país se transforma en un discriminante de igual peso que la condición socioeconómica de los pacientes. Esta injusta asimetría es una deuda para el sistema público de salud y para la comunidad científica que trabaja en cuidados intensivos, quienes están llamados a buscar soluciones concretas y de carácter nacional.

La incompleta integración de los distintos niveles de atención, que afecta la progresión en complejidad de las prestaciones, es determinante en los resultados de las unidades de pacientes críticos, y del uso de recursos involucrados en dichas tareas. La atención inoportuna o inadecuada resolución de los problemas médicos en los niveles básicos de la atención redundará en más demanda de cuidados intensivos y, lo que es peor, condicionará un volumen creciente de sobrevivientes con secuelas significativas, que imponen una carga económica creciente al país y emocional de proporciones a sus familias. Además, la falta de coordinación entre las Unidades de Emergencia de los hospitales de alta 
complejidad (UEH) y sus Unidades de Paciente Crítico (UPC) provoca, entre otros, ingresos tardíos de pacientes mal reanimados, inadecuada utilización de camas críticas en pacientes que debieran acogerse a limitación de terapias con hospitalizaciones prolongadas y altos costos.

Estas asimetrías a lo largo del país y las fracturas de la organización no pueden ser observadas con indiferencia puesto que tienen efectos concretos en la salud de la población. Los problemas estructurales señalados contrastan con el crecimiento de la inversión en salud en Chile, desde 1,9\% del PIB en 1987 hasta 6,7\% en 2012 y en el área de críticos desde $\$ 717.795$ millones hasta $\$ 4.016 .977$ millones, o sea cinco veces más ${ }^{1}$, creando con ello un aumento efectivo de camas críticas (UCI y UTI) desde 773 camas hasta 1.270 camas en los últimos 10 años, aún insuficiente para el aumento de la población y su complejidad. La disponibilidad de estas camas debiera seguir incrementando para lograr el estándar de 6 camas x 100,000 habitantes (considerado un estándar razonable para la realidad del país) y existen nuevos proyectos hospitalarios, en ejecución, que mejorarán la dotación de camas críticas en Chile (Figura 1).

Además está claro que la demanda de este tipo de camas continuará creciendo debido a los cambios demográficos, la mayor expectativa de vida, el incremento en la incidencia de patologías crónicas complejas, siendo los adultos mayores el grupo que más demandará estos recursos. A ello debemos sumar los casos de pacientes que adoptan conductas de riesgo, las condiciones de vida propias de las ciudades modernas y las epidemias intermitentes que afecten al país, todos factores que mantendrán una mayor demanda de camas críticas.

Estamos enfrentando también un período donde existe una relación de mayor simetría entre los pacientes y sus equipos médicos, donde los pacientes, o en su defecto las familias, son protagonistas y gestores de las decisiones de salud que les incumbe, a quienes el Estado además garantiza derechos que ahora son explícitos. La cada vez más judicializada relación médico paciente en una sociedad que ha sido bombardeada sistemáticamente por los medios de comunicación respecto a una medicina "exitista", en que todo parece posible, generan presiones inadecuadas para que pacientes sean admitidos a las UCI para recibir terapias que se comprueban inapropiadas en el corto plazo.

El recurso humano calificado no ha seguido el mismo ritmo de crecimiento que la generación de camas, produciendo con ello una brecha creciente de médicos especialistas y personal clínico en el área, con las consecuencias obvias. Responder a esta demanda es el principal desafío, en especial para el sector público, que hoy, al no tener suficiente capacidad, debe externalizar al sector privado sus pacientes, con costos más elevados Por eso se creó la Unidad de Gestión de Camas Centralizada (UGCC), que es responsable de la adecuada utilización de las camas del sector público, y permite negociar en mejores condiciones la utilización de camas críticas del extrasistema.

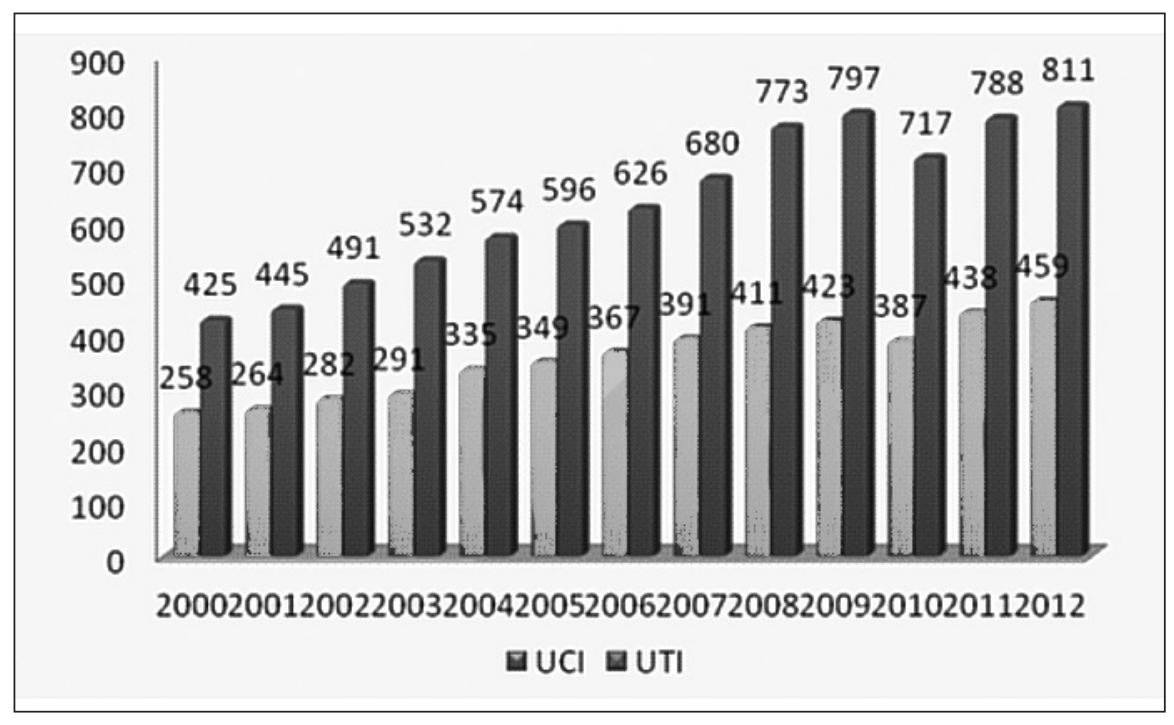

Figura 1. Número de camas críticas adulto ( $\mathrm{UCl}$ UTI) 2000 al 2012. Fuente: Información DEIS. 


\section{Comisión Nacional de Medicina Intensiva del Ministerio de Salud de Chile ${ }^{2}$}

El panorama dado expone al país a un potencial colapso en el área, por falta de médicos interesados en la medicina intensiva. Por estas razones, el Ministerio de Salud de Chile ha convocado a esta Comisión para que busque soluciones factibles de implementar y evitar así un desenlace no deseado para la medicina intensiva en nuestro país.

La Comisión creada en la Subsecretaría de Redes, reúne a representantes de los departamentos universitarios, expertos clínicos del sector público y de la Sociedad de Medicina Intensiva, que analizan globalmente el sector e impulsan iniciativas concretas, como la continuidad de los Seminarios Nacionales, el ciclo de videoconferencias de difusión relevantes para todo el país, buscando afianzar la base que permita a nuestro país contar con una medicina intensiva desarrollada, armónica y equilibrada. Como hito histórico ha implementado el registro de indicadores de calidad equivalentes para todas las UPC, que permitirá realizar un trabajo clínico y de gestión controlado, con fines de pesquisar falencias y brechas para su posterior resolución.

\section{Provisión de especialistas en medicina intensiva}

El plan integral se enfoca en dos ejes prioritarios, en lo que se refiere a la crisis potencial de médicos para las Unidades de Cuidados Intensivos: 1. El déficit real de especialistas en medicina intensiva y la retención de los mismos en el sistema público ${ }^{3}$.

2. En la necesidad urgente de contar con médicos residentes competentes para realizar turnos.

En estas unidades trabajan aproximadamente 860 médicos en sistemas de turnos. La mayoría son especialistas en medicina interna, pocos en anestesiología, menos en cirugía y una minoría son subespecialistas en medicina intensiva. Incluso hay médicos generales que cumplen roles de turno en UCI y persiste un número de cargos no ocupados por falta de interesados, lo que señala el poco interés de profesionales calificados para trabajar en estas unidades. Se evaluó si era factible contar en un plazo razonable con intensivistas para todas las UCI del país. La Comisión llegó a la conclusión de que, dada la velocidad de formación de los especialistas, sólo recién después de varias décadas sería factible contar con estos especialistas para atender todas las UCI, si se mantuviera el actual número de camas críticas en el país, pues se cuenta con un número reducido de centros universitarios (cuatro) que logran formar hoy 10 especialistas cada 2 años, lo que convierte en un objetivo impracticable y poco realista esperar para contar con la presencia de sólo intensivistas en las UCI del país.

De allí, la necesidad de generar alternativas, en las que los especialistas deberían desempeñar un rol como jefes o supervisores técnicos, asegurando la continuidad terapéutica, responsables de guiar al grupo de médicos residentes. El sistema de contrataciones actuales en modalidad de sistema de turnos (cargos de 28 h, Ley 18950), está diseñado para responder a las necesidades de emergencias con pacientes en tránsito; en contraste, en las UCI los pacientes permanecen más de un día. El sistema de turnos provoca una visión fragmentaria de la evolución de los pacientes, que ha sido reconocido por todos los expertos como inadecuado para las tareas del intensivo. De allí la necesidad de dar una visión integradora, por un especialista y que mantenga la coherencia del manejo de los casos. De otro modo, seguirá ocurriendo lo que sucede hoy en muchos lugares, en que las terapias son interrumpidas y fragmentarias, desestabilizando uno de los pilares del éxito en la terapia intensiva, que es justamente la coherencia terapéutica.

La formación de los médicos especialistas está definida por los criterios de acreditación según la Corporación Nacional de Certificación de Especialidades Médicas (CONACEM) y APICE y esta Comisión estima innecesario cambiar esas definiciones. Sin perjuicio de ello, creemos importante un programa de planificación para potenciar la especialidad en el país, precisamente con el perfil de un liderazgo técnico, incorporando incentivos claros y precisos para poder realmente impactar en los desenlaces clínicos y de gestión en dichas unidades.

La necesidad de médicos especialistas (intensivistas) según la recomendación de esta Comisión, se basa en el número de camas críticas de cada unidad ( 1 cama UCI equivale a 2 camas intermedio $[\mathrm{UTI}])$ :

1. En las UCI pequeñas $(<8$ camas UCI o equivalente), bastaría con un intensivista Jefe de Servicio. 
2. En las UCI medianas, grandes o Unidades de Paciente Crítico, se requiere un intensivista Jefe de Servicio, más 1 intensivista como supervisor técnico por cada 12 camas de intensivo, y/o por cada 24 camas de intermedio.

La necesidad de estos especialistas acreditados, a la luz de esta relación, establece una brecha de 75 especialistas y con las expansiones programadas para los años venideros llegará probablemente a 100 especialistas en el sector público ${ }^{5}$.

La Comisión ha incentivado a las autoridades a asegurar la formación de estos especialistas por un programa financiado por el Ministerio de Salud y desarrollado en las cátedras actuales de medicina intensiva. De este modo se cubriría la necesidad estimada en el plazo de 5 a 6 años, evitando una crisis previsible. Estos especialistas, formados con financiamiento del Estado, deberían cumplir al menos el doble de su tiempo (es decir, 4 años) en una UCI/UPC de destinación establecida al inicio de su formación.

Sin embargo, la limitante actual no son los cupos para formación sino los incentivos para que los especialistas se interesen por los programas de formación en medicina intensiva y para que se proyecten como tales en el sector público (sólo una parte de los médicos formados en los programas regulares se desempeña actualmente en el sector público). Para que la formación como intensivista sea una opción atractiva y con el fin de que el sector público pueda retener a los intensivistas formados, esta Comisión hizo presente la necesidad de mejorar significativamente los incentivos económicos, resolver los problemas estructurales y de la organización.

\section{Provisión de médicos residentes, en medicina intensiva}

Se han analizado todas las opciones para asegurar la formación de los médicos residentes, asumiendo que no es indispensable que éstos sean especialistas en medicina intensiva y que es posible buscar otras alternativas para garantizar una masa suficiente de médicos entrenados para cubrir los sistemas de turnos de todo el sistema. La primera consideración para alcanzar dicho objetivo, es el grupo fuente de médicos para ser incorporados en cualquier proceso de capacitación; está claro que partir de la base de médicos recién recibidos representa un universo potencial de más de 1.500 médicos anuales, de donde es posible reclutar interesados. Restringir la búsqueda sólo a médicos especialistas reduce drásticamente las posibilidades a no más de 200-300 médicos por año, además, estos profesionales ya tienen una formación y sus intereses están generalmente determinados y no necesariamente coinciden con las necesidades o prioridades de las UCI.

Luego de los análisis de ventajas y desventajas, la Comisión se ha inclinado por la capacitación de médicos residentes en ciclo de destinación. Este médico tendría competencias mínimas exigibles ${ }^{4}$ para realizar turnos de residencia en una UCI, habilitado por un proceso de adiestramiento en práctica de carácter nacional, directamente dependiente del Ministerio de Salud de Chile, que duraría 2 años de formación sin previa especialidad; o sea, incluye expresamente a médicos recién recibidos de las universidades. Este adiestramiento en práctica, se basa en la formación de competencias, al igual que el Programa CoBaTriCE ${ }^{6}$ de la Sociedad Europea de Medicina Intensiva, que permite asegurar un número de competencias suficientes para el desempeño en sistema de turnos. Este ciclo, similar a médicos generales de zona, permitiría al médico iniciar el proceso y acumular puntaje para acceder a una especialidad formal de su preferencia, luego de haber servido por un período determinado en los hospitales a los que sean destinados. Este programa de adiestramiento en práctica no modifica ninguno de los criterios actuales de la especialidad y se diferencia de otras iniciativas que claramente no cuentan con el consenso de la comunidad de médicos intensivistas.

El número estimado de residentes en ciclo de destinación que deberían formarse para cubrir las necesidades de los turnos de los centros hospitalarios públicos, es de aproximadamente 216 médicos a formar en un lapso de 2 años.

Los residentes en ciclo de destinación estarían obligados a devolver el doble del tiempo de capacitación (4 años) en una UCI a la que haya sido referido desde el inicio del proceso y durante ese período, dependiendo de la ruralidad y el desempeño de la misma, acumularía puntos para optar a una beca de retorno en una especialidad básica. El haber trabajado en el área de críticos les daría un puntaje adicional para postular a las becas primarias. Este modelo ofrece estabilidad al 
sistema, al menos durante un período de 6 años, y permitiría alcanzar una masa suficiente de médicos competentes en un plazo razonable para asegurar la atención de camas críticas a nivel nacional.

Ha existido debate sobre el riesgo relacionado con los médicos incorporados a esta opción, que aún no son especialistas, pues estarían más expuestos a errores y problemas médico legales durante su práctica. Si bien esto no es descartable, lo anterior dependerá en gran medida de cómo sea organizado el adiestramiento, su rigurosidad y si los especialistas responsables mantienen control sobre estos residentes durante la práctica clínica y los turnos. Con ello, es probable que estos problemas sean aminorados; además, se trata de formar un segundo o tercer residente en turno, lo que supone que siempre habrá alguien a quien solicitar ayuda en lo inmediato. Por otra parte, es evidente que esta opción, lejos de ser perfecta o ideal, es menos dramática que seguir teniendo un déficit operacional de camas que irá en aumento y que pone en riesgo un colapso del sistema por falta de profesionales calificados.

\section{Conclusiones}

Los resultados esperados por la Comisión Ministerial son:

$1^{\circ}$ Lograr una red nacional de unidades de pacientes críticos integrados en una organización óptima, con infraestructura moderna, bien equipadas para sus tareas y con personal profesional suficiente, competente y centrado en el paciente.

$2^{\circ}$ Estandarizar a nivel del país las unidades de pacientes críticos, en infraestructura, equipamiento y recursos humanos, conociendo sus indicadores de procesos, resultados y de calidad $y$, de esa manera, transparentar sus brechas en recursos humanos, físicos y de equipamiento.

Asegurar la infraestructura y el equipamiento de las unidades de pacientes críticos sin resolver el problema del recurso médico y profesional de apoyo competente sería un error de proporciones, inaceptable para el nivel que Chile ha alcanzado en esta materia, y que no está en el ánimo de ninguno de los involucrados en esta tarea.

\section{Referencias}

1. Dirección de Presupuestos del Ministerio de Hacienda (DIPRES) http://www.dipres.gob.cl/574/articles-85637_ doc_pdf.pdf

2. Boletín DIGERA $N^{\circ} 4$ Ministerio de Salud de Chile; http://www.minsal.gob.cl/portal/url/item/c27740f33d58a40be0400101640118d5.pdf

3. CANMEDS 2005 Framework Royal College of Physicians and Surgeons of Canada. http://www.royalcollege. $\mathrm{ca} /$ portal/page/portal/rc/common/documents/canmeds/ resources/publications/framework_full_e.pdf

4. La Comunicación de la Comisión al Consejo y al Parlamento Europeo, de 10 de mayo de 2006, núm. 208, para cumplir la Agenda de Modernización para las Universidades; Educación, Investigación e Innovación, Estrategia de Lisboa. http://europa.eu/legislation_summaries/education_training_youth/lifelong_learning/ c11090_es.html

5. Comisión Nacional de Medicina Intensiva del Ministerio de Salud de Chile 2012 http://www.minsal.gob.cl/portal/ url/page/minsalcl/g_varios/plan_invierno/videoconferencias_cnmi.html

6. CoBaTriCE - ESICM 2008. http://www.cobatrice.org/en/ index.asp 